\title{
Coherent emission from a disordered organic semiconductor induced by strong coupling with surface plasmons
}

\author{
S. Aberra Guebrou, ${ }^{1}$ C. Symonds,${ }^{1}$ E. Homeyer,${ }^{1}$ J.C. Plenet, ${ }^{1}$ \\ Yu.N. Gartstein, ${ }^{2}$ V.M. Agranovich, ${ }^{3,4}$ and J. Bellessa ${ }^{1, *}$ \\ ${ }^{1}$ LPMCN; Université de Lyon; Université Lyon 1 and CNRS, UMR 5586; F-69622 Villeurbanne, France \\ ${ }^{2}$ Physics Department, University of Texas at Dallas, Richardson, Texas 75080, USA \\ ${ }^{3}$ Chemistry Department, University of Texas at Dallas, Richardson, Texas 75080, USA \\ ${ }^{4}$ Institute of Spectroscopy, Russian Academy of Sciences, Troitsk, Moscow Region, 142190, Russia
}

(Dated: October 30, 2018)

\begin{abstract}
In this Letter we show that the strong coupling between a disordered set of molecular emitters and surface plasmons leads to the formation of spatially coherent hybrid states extended on macroscopic distances. Young type interferometric experiments performed on a system of J-aggregated dyes spread on a silver layer evidence the coherent emission from different molecular emitters separated by several microns. The coherence is absent in systems in the weak coupling regime demonstrating the key role of the hybridization of the molecules with the plasmon.
\end{abstract}

Localized and delocalized surface plasmon (SP) modes feature stronger confined electric fields and can therefore efficiently interact with molecules or semiconductors close to metallic interfaces [1, 2]. This effect has been widely used to modify optical properties of different types of emitters [3] resulting in the radiative rate enhancement and strong coupling. Another important but less studied aspect is the interaction between different emitters induced by SPs. Efficient energy transfer between donor and acceptor molecules on opposite sides of metal films has been demonstrated [4], the transfer being mediated by symmetric and antisymmetric SP modes on a metallic film. A cooperative emission, similar to Dicke superradiance, of an ensemble of dipoles in the vicinity of a metallic nanosphere has also been theoretically identified [5].

The strong coupling regime offers an interesting perspective for molecular coupling [6] and collective emission. This regime has been reported in various disordered materials such as aggregated dyes coupled to a cavity photon [7] and organic materials [8 11] and semiconductor quantum dots [12] interacting with SPs. Despite the difference of electronic properties of the emitting species in these systems, they all can be seen as a set of localized (no well-defined wavevector) emitters coupled to a propagating extended mode. The strong interaction between a disordered medium and an electromagnetic wave in microcavities has been shown theoretically [13] to result in hybrid eigenstates that are quantum superpositions of a photon and excitations on a large number of molecular sites. Experimentally, the strong coupling is usually identified via observations of an anticrossing in the dispersion relation. The dependence of the Rabi splitting on the concentration of emitters is a good indication of collective effects, however the coherence of spatially remote emitters induced by hybridization with a plasmon (or cavity) mode has never been directly evidenced. In fact, as was theoretically illustrated [14, 15], disorder may result in relatively small modifications of the energetic spectrum of hybrid states, polaritons, while drastically affecting the extent of the polariton wavefunction.

In this Letter we specifically address the issue of spatial coherence and find experimental evidence that the strong interaction between a set of molecular emitters and a SP can lead to the formation of a macroscopic extended coherent state akin to that in a macroscopic polymer chain [16]. We investigate the diffusion and the spatial coherence of the emission of J-aggregated dyes on silver with Young-type interferometric experiments, evidencing an in-phase emission of localized emitters separated by several microns. The extension of such coherent states over a large number of molecules can conceivably be of significance for long-range energy transfer as well as for new optical alloys where the properties of different kinds of emitters spatially separated on a metal film would be mixed via the plasmon.

Our experiments were performed using a leakage radiation (LR) microscopy setup [17], presented in Fig. 1(a). The samples are excited with a laser light at $532 \mathrm{~nm}$ focused on the top side of the sample. The LR of the emission through the silver layer is collected with an immersion oil microscope objective $(\mathrm{NA}=1.49)$ placed in contact with the bottom side of the sample, and imaged either on a CCD camera or on a CCD detector associated with a spectrometer. A notch filter at $532 \mathrm{~nm}$ suppresses the laser radiation in the images. The LR microscopy technique allows a Fourier space imaging providing the angular dispersion of the radiated emission [18] as well as a direct imaging of the emission. In this latter case, a beam block can be inserted in the Fourier plane of the collection optics in order to filter the radiated wavevectors, enabling a selective imaging of the SPs propagating in a given direction. During this study, the experiments were conducted by collecting either all the wavevector components of LR ( $\mathrm{k}_{\text {total }}$ configuration), or solely the wavevector components corresponding to a propagation in the upper half-space as shown in the inset of Fig. 1(a) 


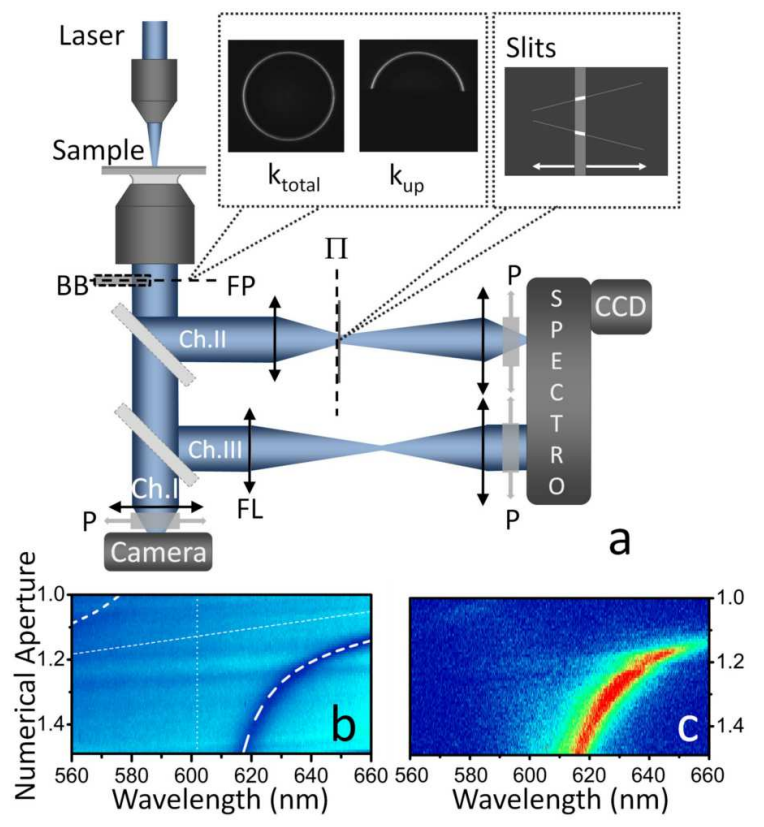

FIG. 1: (a) Experimental setup. A direct imaging is performed on the channel I. The channel II is devoted to coherence measurements by inserting slits in an intermediate image plane $\Pi$ of the sample surface. The channel III is used for dispersion measurements, by imaging the Fourier plane of the microscope objective on the entrance slit of the spectrometer. A beam block (BB) can be inserted in the Fourier plane (FP) to select a given wavevector direction for all the channels. (b) Reflectometry and (c) Luminescence spectra of a TDBC dye layer on a silver film (sample C), displayed as a function of the wavelength and of the numerical aperture (channel III). The doted lines correspond to the bare plasmon and exciton wavelengths, and the dashed line to the calculated polaritonic dispersion.

\section{( $\mathrm{k}_{\text {up }}$ configuration).}

Coherence of the electric field pattern on metal surfaces is associated with highly-delocalized surface modes. This coherence is related to the spatial propagation of the SP but not to the coupling as such between different emitters. This phenomenon has been observed in the infrared range 19] for thermal emitters but can also be present in the optical range [20]. In order to clearly evidence the effect of the SP-exciton hybridization, samples in the weak and strong coupling regimes have been compared.

Three samples were investigated, each elaborated by spin coating an active layer onto a $45 \mathrm{~nm}$ silver film thermally evaporated on a glass coverslip. The active layer of the first sample (sample A) is composed by widely separated fluorescent polystyrene microspheres, emitting at $560 \mathrm{~nm}$ and having a diameter of $100 \mathrm{~nm}$. The distance between each microsphere is sufficient to consider the emission of an insulated nanoparticle. The second sample (sample B) contains a continuous layer of CdSe quantum dots emitting around $660 \mathrm{~nm}$. The distance between the quantum dots is smaller than the resolution of our setup,

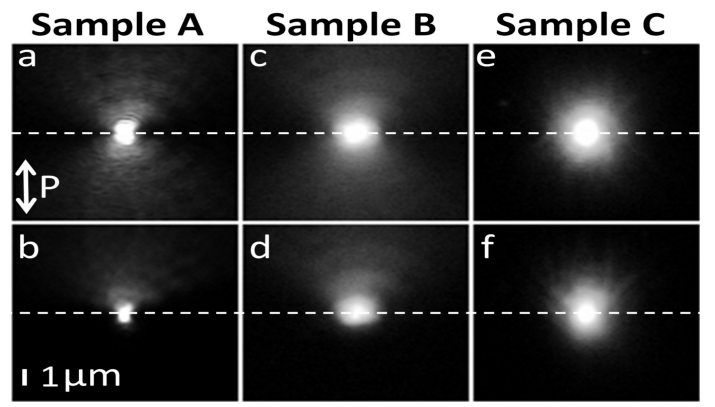

FIG. 2: Direct images of the leakage radiation on the surface for the different samples: (a) and (b) sample A (insulated microspheres). (c) and (d) sample B (CdSe nanocristals). (e) and (f), sample $\mathrm{C}$ (TDBC layer in strong coupling). Panels (a), (c) and (e) correspond to the $k_{\text {total }}$ detection and panels (b),(d) and (f) to $k_{u p}$ detection configuration. The vertical arrow indicates the polarization direction $\mathrm{P}$. The horizontal dotted lines correspond to the position of the emission center.

so that an uniform emission pattern will appear on the images. From the dispersion relations obtained by reflectometry measurements on these two samples, it has been checked that both kind of emitters are in weak coupling regime with the SP (data not shown) 21]. In the third sample (sample $\mathrm{C}$ ) the optically active component is a J-aggregated 5, 5', 6, $6^{\prime}$ - tetrachloro - 1, $1^{\prime}$ - diethyl $3,3^{\prime}-$ di $(4-$ sulfobutyl $)$ - benzimidazolo-carbocyanine (TDBC). The TDBC layer is formed by an ensemble of linear J-aggregates chains with a small length compared to the wavelength, and thus can be seen as a set of independent emitters randomly spread throughout the film. Figure 1(b) presents the reflectometry experiment recorded in the Fourier space for sample $\mathrm{C}$. The measured dispersion relation presents an anticrossing characteristic of a strong coupling regime between the SP and the TDBC exciton, as previously reported for this kind of sample [8]. The Rabi splitting energy calculated with a classical two level oscillator model is $300 \mathrm{meV}$.

In a first set of experiments the emission through the silver layer for each sample was imaged on the CCD camera. For the sample $\mathrm{A}$ in the $\mathrm{k}_{\text {total }}$ configuration (Fig. 2(a)), the emission of one microsphere forms two lobes oriented in the vertical direction. When the beam block is inserted in the Fourier plane $\left(\mathrm{k}_{u p}\right)$, only the radiation having wavevector components in the upper halfplane is detected. In this case the lower emission lobe is suppressed (Fig. 2(b)). These images can be clearly interpreted in terms of a localized particle emitting SPs propagating upwards and downwards (Fig. 2(a)) or only upwards (Fig. 2(b)). The same behavior is observed for sample B (Fig. 2(c),(d)). In these images the bright central area corresponds to a collection of CdSe quantum dots excited by the laser beam. The emission pattern presents two lobes, the lower one being suppressed in $\mathrm{k}_{u p}$ configuration (Fig. 2(d)). The set of emitters in the 
weak-coupling regime with SP modes behaves as a sum of independently emitting particles. The recorded emission patterns are drastically different when the emitters are in the strong-coupling regime with SPs, exhibited by sample C (Fig. 2 (e),(f)). In this case, the emission pattern has an oval shape in the $\mathrm{k}_{\text {total }}$ configuration and is only slightly modified in the $\mathrm{k}_{u p}$ configuration. The persistence of the lower lobe in the $\mathrm{k}_{u p}$ configuration indicates that the emission can not be interpreted in terms of a set of particles which independently emit propagating SPs but rather as arising from an ensemble of emitters all coupled to the same SP resulting in extended hybridized polaritonic states.

In order to study the spatial coherence of this emitter assembly, two Young's slits are inserted in an intermediate image plane of the sample. The Youg slits are obtained by crossing a vertical slit with two $\mathrm{V}$ shaped slits in order to vary the interslit distance. The resulting slits, located on both sides of the excitation spot (full width at half maximum FWHM $0.7 \mu \mathrm{m}$ ), select the emission from two regions of the sample separated by a distance of $2.8 \mu \mathrm{m}$. The interference pattern recorded from sample A (a single localized emitter) is shown in Fig. 3(a). Interference fringes appear on the image. This case is analogous to a classical wavefront division interference experiment: the emitter generates SPs propagating upwards and downwards before reaching the spatial regions selected by the upper and lower slits. The radiation propagating along these two paths interferes on the entrance slit of the spectrometer. In the $\mathrm{k}_{u p}$ configuration, however, which disables the lower direction of propagation, only the upper slit remains illuminated and the interference fringes disappear (Fig. 3(b)). In the case of sample $\mathrm{B}$ no fringes are visible in both configurations. This is what is expected for a set of independent localized emitters. Each of them generates its own interference pattern associated with a phase difference wich depends on its position between the slits. As the excitation spot extends over $0.7 \mu \mathrm{m}$, a blurring of the fringes occurs.

In contrast to samples $\mathrm{A}$ and $\mathrm{B}$, sample $\mathrm{C}$ displays interference patterns in both $\mathrm{k}_{\text {total }}$ (Fig. $3(\mathrm{e})$ ) and $\mathrm{k}_{u p}$ (Fig. 3(f)) configurations between $610 \mathrm{~nm}$ and $645 \mathrm{~nm}$, corresponding to the SP-exciton mixed (polaritonic) states. The persistence of the interference fringes, even when the SP-assisted interferences are suppressed $\left(\mathrm{k}_{u p}\right)$, demonstrates the spatial coherence of the extended hybrid states over a distance of $2.8 \mu \mathrm{m}$. The results may be compared to similar experiments performed on long polymer chains [16]. For those long molecules the emission along the chain is coherent: the whole polymer chain emits even when excited with a localized spot. In a comparable manner, in our case a single dye chain can not be excited independently from the others as shown in the diffusion experiments of Fig. 2.

The spatial extent of the SP-exciton hybrid states is now further addressed with a similar Young experiment

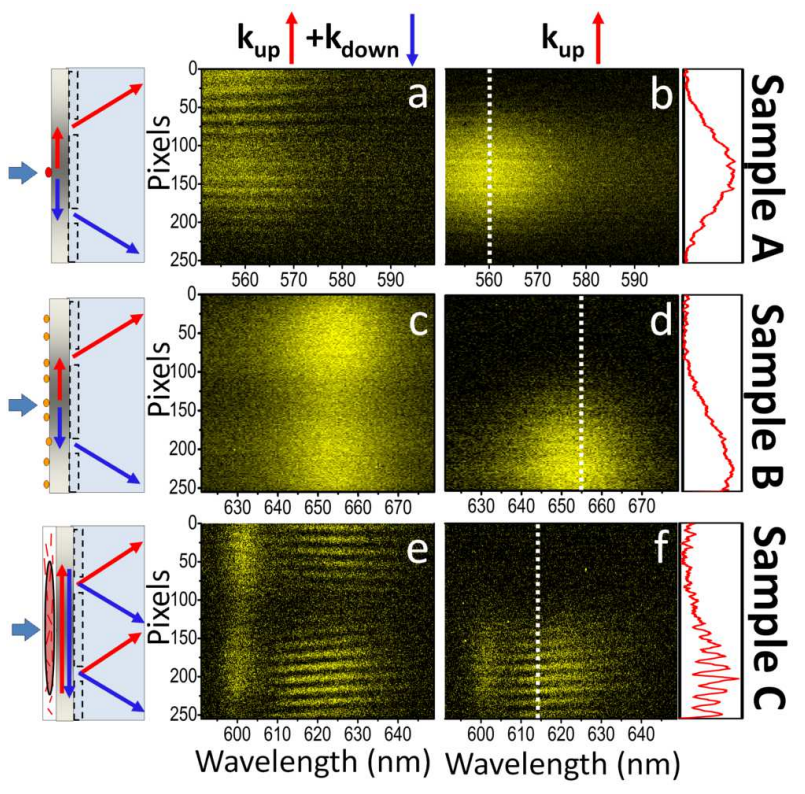

FIG. 3: Interference pattern recorded for samples A, B and C: without selection on the wavevector (panels (a), (c) and (e), respectively) and with only the upwards propagation (panels (b), (d) and (f), respectively). Layouts of different propagation mechanisms are drawn on the left side of the figure for each of the samples. For the $\left(\mathrm{k}_{u p}\right)$ configuration, the intensity profile measured along the white dotted line is drawn on the right side of the figure.

but with a laser spot (FWHM $10 \mu \mathrm{m})$ covering both interfering regions on the sample. The emission pattern of a control sample consisting of a TDBC layer directly deposited on a glass cover slip without silver is shown in Fig. 4(a) and does not display any interference fringes, which agrees with the assertion that the TDBC layer is constituted by independent emitters formed by the aggregated dye chains. Interference images are recorded for sample C, TDBC layer on silver (Fig. 4(b)). Two different regions can be seen in the image: the polaritonic emission between 610 and $645 \mathrm{~nm}$ presenting interference fringes and the emission around $600 \mathrm{~nm}$ without interferences. The latter emission comes from incoherent states at the bare TDBC exciton energy. The existence of both incoherent and coherent extended states in the polariton spectrum has been discussed theoretically [13 15$]$ for organic microcavities in the strong-coupling regime. Fig. 4(c) presents the visibility of the fringes as a function of the distance between the interfering regions. For a detection wavelength of $630 \mathrm{~nm}$, the visibility vanishes at $7.5 \mu \mathrm{m}$, while for $610 \mathrm{~nm}$ the visibility decreases faster and vanishes around $6.5 \mu \mathrm{m}$ showing a reduction of the coherence length when the wavelength becomes closer to the bare exciton emission at $600 \mathrm{~nm}$.

To elucidate the generic character and the origin of the frequency-dependent degree of coherence, we now provide an illustrative calculation of surface polaritonic states in 


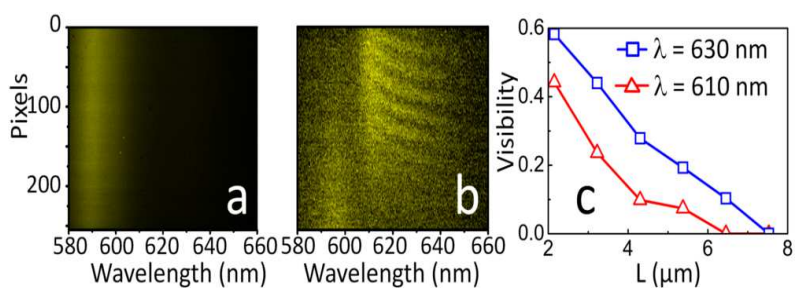

FIG. 4: (a) Emission pattern for a TDBC layer directly deposited on a glass substrate. (b) Typical interference pattern for a TDBC layer on silver with an excitation spot larger than the interslit distance (here $4.4 \mu \mathrm{m}$ ). (c) Visibility of the fringes as a function of the distance for two detection wavelengths (610 and $630 \mathrm{~nm}$ ).
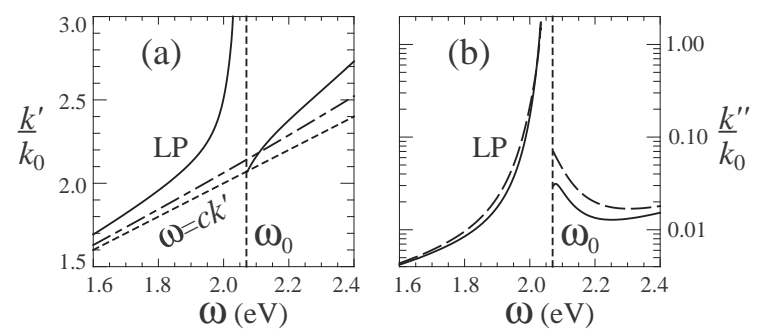

FIG. 5: Dispersion of surface polaritons in the vicinity of the resonance with the excitations of the thin layer: (a) Real and (b) Imaginary parts of the wave vector as a function of frequency in units of $k_{0}$, the wave number of the vacuum photon at $1 \mathrm{eV}\left(\lambda_{0} \simeq 1240 \mathrm{~nm}\right)$. The Rabi splitting of two polariton branches is clearly seen in panel (a), which also displays the bare (in the absence of the layer) SPs by a dashdotted line. Numerical parameters used: $\omega_{0}=2.07 \mathrm{eV}, \varepsilon_{0}=$ $3.05, d=20 \mathrm{~nm}, A=2 \mathrm{eV}^{2}, \delta_{0}=14 \mathrm{meV}, \varepsilon_{2 b}=3.29$, $\omega_{p}=8.93 \mathrm{eV}, \delta=79 \mathrm{meV}$. Dashed lines in panel (b) show the results for larger $\delta_{0}=25 \mathrm{meV}$.

a simpler parent system consisting of a thin (thickness d) resonating organic layer on a metallic substrate [22]. The dispersion equation (DE), frequency $\omega$ vs in-plane wave vector $k$, can be found in this case as

$$
\kappa_{1} / \varepsilon_{1}+\kappa_{2} / \varepsilon_{2}=\beta\left(k^{2} / \varepsilon_{\perp}-\omega^{2} / c^{2}+\varepsilon_{\|} \kappa_{1} \kappa_{2} / \varepsilon_{1} \varepsilon_{2}\right),
$$

where $\kappa_{i}^{2}=k^{2}-\varepsilon_{i} \omega^{2} / c^{2}, \beta=-\tanh (\kappa d) / \kappa$ and $\kappa^{2}=\left(\varepsilon_{\|} / \varepsilon_{\perp}\right) k^{2}-\varepsilon_{\|} \omega^{2} / c^{2}$. The DE features various $\omega$-dependent dielectric functions, their imaginary parts being representative of the dissipation and disorder in the system. In the absence of the layer, $\beta=0$, the DE would yield standard SP excitations at the interface between dielectric (we choose vacuum $\varepsilon_{1}=1$ ) and metallic $\left(\varepsilon_{2}=\varepsilon_{2 b}-\omega_{p}^{2} / \omega(\omega+i \delta)\right)$ half-spaces. The organic layer here is anisotropic: for layered $\mathrm{J}$-aggregates we may assume that the resonance (frequency $\omega_{0}$ ) occurs only for the the polarization along the layer $\left(\varepsilon_{\|}=\right.$ $\left.\varepsilon_{0}+A /\left(\omega_{0}^{2}-\left(\omega+i \delta_{0}\right)^{2}\right)\right)$, while no resonance polarization takes place perpendicular to the layer $\left(\varepsilon_{\perp}=\varepsilon_{0}\right)$.

Fig. 5 shows the resulting two polaritonic branches in the form of the real and imaginary parts of the wavevec- tor $\left(k=k^{\prime}+i k^{\prime \prime}\right)$ as functions of frequency $\omega$ in the vicinity of the resonance $\omega_{0}$. Displayed are the states beyond the light line $\left(\omega<c k^{\prime}\right)$, which would be stationary states $\left(k^{\prime \prime}=0\right)$ in the absence of the dissipation in the metal $(\delta=0)$ and the layer $\left(\delta_{0}=0\right)$. It is the non-vanishing dissipation (disorder) that makes these states decaying, the decay length $\left(1 / k^{\prime \prime}\right)$ establishing the spatial scale of the coherence. We attribute the experimentally observed features to the behavior exhibited by the lower-energy polaritons (LP) in Fig. 5 where the decay length can indeed be in the $\mu \mathrm{m}$ range below the resonance frequency (e. g., $\sim 2.2 \mu \mathrm{m}$ for $\lambda=625 \mathrm{~nm}$ in this example) but strongly decreases upon the approach to the resonance. This trend agrees with observations in Fig. 4.

The formation of macroscopic coherent states is expected for a large number of disordered materials like organic dyes [8, 11], layers of quantum dots [12], and rare earth ions [23] strongly coupled to SPs. The simple design of the samples enables the manipulation of these coherent states by structuring the active layer at distances shorter than the coherence length, using patterns or inclusion of several different materials, opening a way to a new class of plasmonic materials.

The authors thank J. Bloch, R. Grousson and M. Broyer for helpful discussions and support. This work has been supported by the French ANR PNANO SCOP and by the Lyon Nanoptec center. V.M.A. acknowledges hospitality of Scuola Normale Superiore (Pisa) via support of the European Commission (Grant N. FP7-PEOPLEITN-2008-237900 "ICARUS") and thanks Russian Foundation for Basic Research for partial support.

* Electronic address: joel.bellessa@univ-lyon1.fr

[1] A. V. Akimov et al., Nature 450, 402 (2007).

[2] Y. Fedutik et al., Phys. Rev. Lett. 99, 136802 (2007).

[3] P. Anger, P. Bharadwaj and L. Novotny, Phys. Rev. Lett. 96, 113002 (2006).

[4] P. Andrew and W.L. Barnes, Science 306, 1002-1005 (2004).

[5] V.N. Pustovit and T.V. Shahbazyan, Phys. Rev. Lett. 102, 077401 (2009).

[6] D.G. Lidzey et al., Science 288, 1620-1623 (2000).

[7] D. G. Lidzey et al., Phys. Rev. Lett. 82, 3316 (1999).

[8] J. Bellessa et al., Phys. Rev. Lett. 93, 036404 (2004).

[9] J. Dintinger et al., Phys. Rev. B 71, 035424 (2005).

[10] Y. Sugawara et al., Phys. Rev. Lett. 97, 266808 (2006).

[11] T. K. Hakala et al., Phys. Rev. Lett. 103, 053602 (2009).

[12] D.E. Gomez et al., Nano Lett. 10, 274-278 (2010).

[13] V.M. Agranovich, M. Litinskaia and D.G. Lidzey, Phys. Rev. B 67, 085311 (2003).

[14] V.M Agranovich and Y.N. Gartstein, Phys. Rev. B 75, 075302 (2007).

[15] M. Litinskaya and P. Reineker, Phys. Rev. B 74, 165320 (2006).

[16] F. Dubin et al., Nature Phys. 2, 32-35 (2006).

[17] B. Hecht et al., Phys. Rev. Lett. 77, 1889-1892 (1996). 
[18] A. Drezet et al., Appl. Phys. Lett. 89, 091117 (2006).

[19] R. Carminati and J.J. Greffet, Phys. Rev. Lett. 82, 16601663 (1999).

[20] R. Zia \& M.L. Brongbergsma, Nature nano. 2, 426-429 (2007).

[21] It has to be noted that the CdSe quantum dots can switch from weak to strong coupling if their concentration is increased by five orders of magnitude [12]. Nevertheless another material, namely the TDBC, has been chosen for experiments in strong coupling. Indeed, the TDBC allows a Rabi splitting more than 4 times larger than the polariton linewidths, thus ensuring an interaction with plasmon largely prevailing the scattering and damping of the system.

[22] V.M. Agranovich \& A.G. Malshukov, Opt. Comm. 11, 169-171 (1974).

[23] M. Lipson \& L.C. Kimerling, Appl. Phys. Lett. 77, 11501152 (2000). 